\title{
Simple Interventions Improve the Quality of a Missed Lab Appointment Process
}

\author{
Martina Mookadam, Michael Grover, Chris Pullins, Mary Winscott, Susan Pierce \\ Mayo Clinic Arizona, United States
}

\begin{abstract}
Simple interventions resolve the problem of missed lab appointments.

It is essential that patients complete ordered laboratory studies. This maintains clinical quality and, potentially, keeps patients safe from harm. In our academic family medicine practice, baseline data demonstrated patients completed $94.7 \%$ of labs as ordered (26850/28348 patients per year) while $1498(5.3 \%)$ did not. Our baseline patient reminder process, a mail or portal based generic letter, resulted in only 449 (30\%) of patients ultimately completing them (1049 [70\%] did not). Our baseline system was $96.3 \%$ reliable. This process did not allow for provider review or input, and was not personalized for patients.
\end{abstract}

We designed a quality improvement project involving three PDSA (Plan, Do, Study, and Act) cycles of about two months each. Desk staff created weekly reports of unresolved lab orders. A message in the electronic medical record (EMR) solicited provider input. Providers could elect to cancel studies (if already completed, reordered, or no longer clinically indicated) or have the patient receive a personalized reminder, including provider name and associated diagnoses. This reminder was sent by patient portal secure messaging (if an account existed) or with a mailed letter. These interventions resulted in $98.8 \%$ process reliability. The frequency of unresolved lab orders decreased from $70 \%$ at baseline to $25 \%$.

In the second PDSA cycle, we contacted patients by the portal only if there was evidence of an active account. Otherwise, they were contacted by telephone. Patients without a portal account continued to receive a letter by mail. These modified processes resulted in an overall reliability rate of $99.2 \%$. The frequency of unresolved lab orders decreased to $17 \%$.

A final PDSA cycle utilized only telephone contact with patients with unresolved lab orders. Schedulers offered patients a choice of appointment dates if they spoke personally. Otherwise, they were left messages with a future lab appointment date two weeks later. Overall process reliability now increased to $100 \%$. The frequency of unresolved lab orders decreased to $0 \%$.

Our interventions resulted in increased system reliability. Provider input was not perceived as burdensome. Desk staff work effort was not increased. Telephone patient contact resulted in more frequent lab order completion than other methods.

\section{Problem}

A group of Family Medicine physicians working within an academic integrated multidisciplinary medical group practice in Scottsdale, Arizona noted that some patients were missing their scheduled laboratory appointments. These appointments had been ordered by their primary care providers for follow up of acute or chronic medical conditions. Missed laboratory appointments (defined here as blood and urine studies) place patients at risk for adverse outcomes related to incomplete laboratory follow evaluation.

\section{Background}

Missed lab appointments can go silently ignored if there is no system in place to make the clinician aware. Reported studies show that a significant percentage of patients fail to complete lab appointments as ordered.[1] These instances can potentially create otherwise preventable sentinel events in the lives of patients or allow chronic disease to progress unchecked.[2,3] Additionally, patients who missed lab appointments can be viewed negatively by primary care staff[2] which can impact therapeutic relationships. While the utilization of a robust electronic medical record can aid in capturing these missed appointments and provide the clinician opportunity to safely close the loop, our literature search revealed very few papers addressing effective interventions.

\section{Baseline measurement}

For this project, three baseline measurements were performed. First we identified the total number of lab appointments ordered over a 12 month period from September 2013 through August 2014 by family medicine physicians and nurse practitioners. The second measure was the number of patients who failed to come in for missed appointments over the same time period and the third was the number of patients who were successfully rescheduled as part of the system that was already in place. 
Baseline data demonstrated patients completed $94.7 \%$ of labs as ordered (26850/28348 patients per year) while 1498 (5.3\%) did not. Our baseline patient reminder process, a mail or electronic portal based generic letter, resulted in only $449(30 \%)$ of patients ultimately completing lab orders while 1049 (70\%) making our baseline system $96.3 \%$ reliable. Additionally, this process did not allow for provider review or input and was not personalized for patients.

\section{Design}

When considering the possible reasons for the current system failure, we decided that the process needed to become more patient-centered. An intervention was designed to communicate the importance of having the labs completed to the patient on a more personal level. A number of different ideas were considered, including having the clinician or their allied health staffs personally call patients, however, these options did not seem sustainable over time. The agreed upon intervention was that each provider would receive a message through our electronic medical record system to advise them that their patient had missed a lab appointment. The provider was prompted to respond to the message and request a) the lab order be cancelled (if deemed to no longer be needed) or b) request a reminder be sent to the patient which included the corresponding diagnosis for the lab study. Reminder letters were sent via the electronic patient portal (internet or mobile application that allows patients 24 hour access to their care team, electronic medical record, and upcoming appointments) or through the US postal service to patients without a portal account. The letters were structured so that they could easily be personalized by inserting relevant information. If after 14 days, there was still no response, a second and final letter was sent to the patient.

The intervention was felt to potentially be sustainable for the following reasons: 1 . There was no extra monetary cost involved 2 . A system was already in place that could be modified and improved. Specifically, missed lab reports were already being generated and systems were in place for contacting patients regarding appointments. 3 . The intervention was of a simple design, easily updatable, automated, and patient centered. 4 . We had provider buy-in and support for the process.

\section{Strategy}

PDSA Cycle 1: Missed lab reports were run weekly. Through our electronic medical record, trained front desk staff sent messages to providers whose patients has missed lab appointments in the preceding week. Providers were given the choice of canceling the labs if no longer needed or requesting a reminder be sent to the patient requesting re-scheduling. When the second option was chosen, the corresponding diagnosis was included. The front desk staff then continued the work flow by either canceling the labs or sending the first personalized letter to the patient. If no response was received after two weeks, a second and final reminder was sent to the patient. After this intervention, we saw some improvement, however, $24 \%$ of missed labs remained unresolved.
PDSA Cycle 2: Missed lab reports were run weekly. Providers were again given the same information and choices. In this cycle, patients were contacted through the portal only if there was evidence of an active portal account. Otherwise, they were contacted by telephone. Patients without a portal account received a letter by mail. This modified process further improved reliability.

PDSA Cycle 3: A final PDSA cycle utilized only telephone contact with patients who had unresolved orders. When direct patient contact was made by telephone, an appointment was made at that time. Otherwise a detailed message was left including the provider's name and associated diagnosis with a future lab appointment being scheduled two weeks later. Overall process reliability increased to $100 \%$.

See supplementary file: ds7435.docx - "Examples of letters sent to patients first PDSA Cycle"

\section{Results}

The aim of our quality improvement project was to increase the reliability of our system to resolve missed lab appointment orders. We designed a quality improvement project involving 3 PDSA cycles of approximately two months each. After initial interventions the frequency of unresolved lab orders decreased from $70 \%$ at baseline to $24.2 \%(45 / 186)$ after the first PDSA cycle. These interventions resulted in an overall reliability of $98.9 \%$. After the second PDSA cycle, modifications in our contact work flow resulted in further reliability improvement to $99.2 \%$ with the frequency of unresolved labs orders decreasing to $16.9 \%$ (59/349). Overall process reliability after the final PDSA cycle improved to $100 \%$ with a frequency of 0/288 lab orders remaining unresolved when patients were contacted via telephone only.

With regard to sustainability, since full implementation of the third PDSA cycle, we continue to have excellent patient compliance with the system remaining almost $100 \%$ reliable $(99.9 \%$ reliable at the most recent three month review).

See supplementary file: ds7524.pdf - "PDSA cycles 1,2,3"

\section{Lessons and limitations}

During implementation of this project, we learned a number of lessons. First, engaging all the stakeholders including physicians and front desk staff was key to determining our baseline process and then implementing the PDSA cycles. We also gained a deeper understanding of how patients interact with the EMR. For example, patients who are sending messages through the portal when they need refills or are seeking advise from us don't necessarily respond to incoming messages from the health care team. The final PDSA cycle confirmed how important personal interactions were for patients with direct telephone contact being better than any other intervention in resolving missed lab appointments.

\section{Conclusion}


We recognized a lack of reliability in our system of managing follow up for patients who missed their laboratory appointments. With that system, $70 \%$ of patients who missed their lab appointments were having no endpoint action completed upon that event. With this, an endpoint action was considered either that patient ultimately had the labs completed or the provider determined that the lab orders could be cancelled. Our intervention involved a patient centered approach that included providers in the follow up plan, resulting in a more personalized approach for the patient. A combination of simple interventions with patient contact by telephone and provider input to renew or cancel labs orders via the EMR allowed for increased system reliability. Provider input was not perceived as burdensome.

Importantly, desk staff work effort was not increased. Prior to initiating the QIR project, there was already a process in place for contacting patients who had missed lab appointments. Desk staff were already spending time running weekly reports and then contacting the patients with letters or portal messages. As the project developed, time spent on the process was comparable and more straight forward with the final process being only a phone call to the patient to reschedule. Reaching out to providers was an extra step however, as some patients did not need to get rescheduled after provider input, this also decreased work effort for the desk staff. As the process has been in place for over a year at this point, no extra desk staff has been required and feedback continues to be positive. Certainly a practice setting where missed labs had not yet been address with a specific process would see an increase in staff effort.

After the third PDSA cycle, we decided it was time to spread the intervention to other departments at our institution. Because of our close physical proximity and because we share front desk staff, we first approached our colleagues in Women's Health. We presented the results of our quality improvement project at their department meeting which included physicians, nurses, and allied health staff involved in scheduling patients. As a result, they have begun to implement the new missed labs work flow. Once we have shown successful implementation within another department, our strategy is to spread the intervention to all of primary care and then potentially to the specialty clinics within our large academic institution. Additionally, we presented our project at a national meeting focusing on the science of health care delivery, encouraging spread of the intervention beyond our own clinics.

\section{References}

1 Callen, Joanne L., Westbrook, Johanna I., Georgiou, Andrew, Li, Julie., Failure to follow-up test results for ambulatory patients: a systematic review. Journal of General Internal Medicine. 2012, 27;10 pp 1334-48.

2 Mahvash Husain-Gambles, Richard D Neal, Owen Dempsey, Debbie A Lawlor, and Jim Hodgson., Missed appointments in primary care: questionnaire and focus group study of health professionals. The British Journal of General Practice. 54;499. Pp108-13
3 Elder, Nancy C., Hickner, John. Missing clinical information: the system is down. JAMA. 2005. Vol: 293 iss:5 pg 617-9

\section{Declaration of interests}

I have read and understood BMJ group policy on declaration of interests and declare the following interests: NONE

\section{Acknowledgements}

We would like to thank Susan Greger MD for her invaluable input in first identifying the problem and helping with the initial project plan.

\section{Ethical approval}

According to the policy activities that constitute research at Mayo Clinic, this work met criteria for operational improvement activities exempt from ethics review. Our project studied the implementation of a practice to improve the quality of patient care, and collection of patient/provider data regarding implementation of the practice for clinical, practical and administrative purposes. As such, our project is considered non-research quality improvement does not require submission to the Institutional Review Board. 